\title{
MODELADO HIDRODINÁMICO DE LA BAHÍA DE CÁDIZ
}

\author{
Carmen Zarzuelo Romero* \\ Tutores: Dr. Miguel Ortega Sánchez y Alejandro López Ruiz \\ Máster Interuniversitario de Hidráulica Ambiental, especialidad de Puertos y Costas. Curso 2011-2012
}

\begin{abstract}
Resumen
Esta tesina fin de máster se ha centrado en la caracterización hidrodinámica de la Bahía de Cádiz, situada al Sur-Oeste de la Península Ibérica, mediante medidas de campo y modelado numérico. Con base en la caracterización morfológica de los sacos de la Bahía de Cádiz, se ha diseñado y realizado recientemente una campaña de campo que duró desde Diciembre del 2011 hasta Mayo de 2012 empleando un total de 13 instrumentos. Asimismo, se ha aplicado y validado el modelo hidrodinámico DELFT3D (módulo FLOW), obteniendo una buena correlación tanto para niveles $(\mathrm{RMSE}=0.98)$ como para las velocidades $(\mathrm{RMSE}=0.9)$. Este modelo se ha empleado para analizar los efectos que el dragado que se realizará para la ampliación del puerto de Cádiz tendrá sobre la hidrodinámica de la Bahía.
\end{abstract}

Palabras clave: Bahía, Cádiz, Hidrodinámica, Medidas de Campo, DELFT-3D

\section{Introducción}

La Bahía de Cádiz está situada entre los municipios de Rota, El Puerto de Santa María, Puerto Real, San Fernando, Chiclana de la Frontera y Cádiz (Fig 1). Geográficamente se encuentra dividida en tres partes: (1) la Bahía Exterior, bañada por aguas atlánticas, (2) la Bahía Interior, rodeada en su totalidad por tierra emergida, (3) y el Saco Central, unión de la bahía interior con la bahía exterior a través del Estrecho de Puntales.

Las actividades antropogénicas en la Bahía de Cádiz y su sistema costero, como el dragado del canal para la ampliación del puerto y la construcción del puente "La Pepa", han eliminado o desplazado grandes cantidades de sedimentos en el último siglo (Gutiérrez-mas et al., 2009; Tejedor, 1997; Alvarez et al., 1999), aunque el impacto de estas perturbaciones en el sistema costero no se ha cuantificado. $\mathrm{El}$ intercambio de flujos de agua, sedimentos y sustancias entre el saco interno y externo de la Bahía de Cádiz es esencial para comprender los cambios observados y desarrollar herramientas para su gestión integrada.

\footnotetext{
*zarzueloc@ugr.es
}

En el informe de sostenibilidad ambiental del plan director del puerto de la Bahía de Cádiz se estudiaron de forma preliminar las posibles modificaciones hidrodinámicas inducidas por la ejecución del dragado. Éstas podrían tener un impacto positivo en la Bahía Interna, ya que la regularización de fondos y márgenes podría mejorar el intercambio de aguas en la Bahía al uniformizar el sistema de corrientes de marea y aumentar el prisma de marea, incrementando la calidad ambiental del entorno. Asimismo, la construcción del nuevo puente puede ocasionar cambios en el intercambio de las masas de agua de la Bahía Externa y la Bahía Interna (Fig. 1) al disminuir el área transversal en la que se produce el intercambio de agua (Estrecho de Puntales) (Fig. 1). La evaluación y cuantificación de los efectos que las actuaciones anteriores pueden producir en la Bahía resultan fundamentales para su gestión integrada a medio y largo plazo, siendo ésta la motivación principal del presente trabajo.

Los estudios previos sobre la hidrodinámica de la Bahía de Cádiz se han centrado en el análisis de las contantes mareales (Álvarez et al., 2003; Kagan et al., 2001, 2003, 2005) y en la influencia de la estacionalidad en la variabilidad mareal (Tejedor, 1997). Se han basado en medidas de campo y modelos 2D no lineales, así como en el estudio de la 


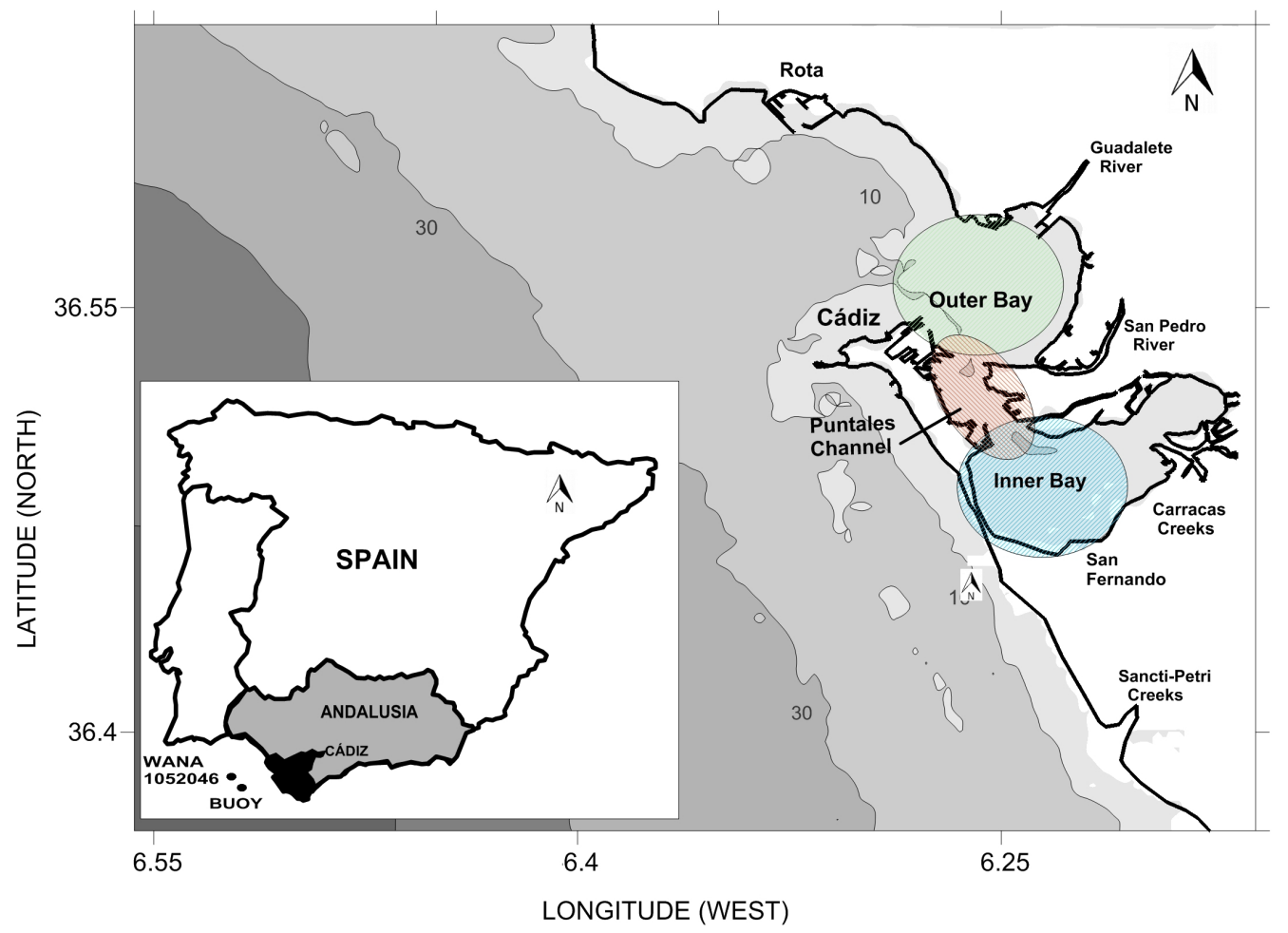

Figura 1: Zona de estudio-Bahía de Cádiz

interacción viento-oleaje/marea. También se ha estudiado la relación que existe entre los sedimentos (Alvarez et al., 1999) y los componentes orgánicos (De la Paz et al., 2008) con la dinámica mareal. Asimismo, durante los últimos años se ha incrementado el estudio de la influencia que los caños mareales, los ríos y sus mutuas interacciones tienen sobre la Bahía (Ferrón et al., 2009; Benavente et al., 2006).

La complejidad hidrodinámica de sistemas costeros como la Bahía de Cádiz requiere estudiar las variaciones espaciales y temporales de los flujos hidrodinámicos. Para el estudio de este tipo de procesos se suelen emplear datos de campo y modelado numérico (Elias, 2006; Lesser, 2009; Van der Wegen et al., 2010). En el marco de algunos estudios sobre la Bahía de Cádiz se han realizado campañas de campo pero con una corta duración. Además no han llegado a profundizar en la hidrodinámica, como por ejemplo en las corrientes, centrándose en otros aspectos como el transporte de sedimentos (Álvarez et al., 2003; Kagan et al., 2003, 2005, 2003). En este trabajo se ha realizado una campaña de campo de 5 meses de duración con el objetivo de mediar datos y caracterizar la variación tanto temporal como espacial de la hidrodinámica a lo largo de la Bahía de Cádiz.

Van der Wegen et al. (2010), Lesser (2009) y Elias and Hansen (2012) han mostrado la utilidad de emplear modelos numéricos para el estudio a medio y largo plazo de procesos hidrodinámicos en entornos costeros similares a la Bahía de Cádiz. Asimismo, el estado del arte actual permite también el modelado hidrodinámico y morfológico en el corto plazo, analizando así la influencia de los diferentes procesos físicos. Las especificidades de la zona de estudio sugieren del uso de modelos 3D (Tejedor, 1997), cuyo desarrollo se ha incrementado significativamente durante los últimos años. Por ello, en este trabajo se ha usado el modelo DELFT3D que actualmente es de distribución gratuita. Se trata de un modelo que consta de distintos módulos integrados que permiten la simulación de la hidrodinámica en zonas de geometría compleja (Lesser et al., 2004).

La finalidad de este artículo es presentar la campaña de campo realizada recientemente y los primeros resultados del estudio combinado datos de campo - modelo numérico. Así, este trabajo se centra en la caracterización morfológica de los sacos, 
en el diseño y realización de la campaña de campo, en el análisis preliminar de una parte de los datos y en la implementación y validación del modelo hidrodinámico.

\section{Zona de estudio}

\subsection{Geografía}

La Bahía de Cádiz está situada al Sur-Oeste de España, con una extensión de 140 km2 aproximadamente desde el interior hasta Rota (Fig. 1). En ella desembocan los ríos Guadalete, Iro y Salado de Rota. Otros dos hitos a resaltar en esta zona son el caño de Sancti Petri y el río San Pedro. En su entorno, además, se pueden destacar las marismas, esteros y el Parque Natural de la Bahía de Cádiz, protegido jurídicamente.

La zona de estudio presenta gran complejidad debido a la confluencia e interacción de la marea (astronómica y meteorológica), la dinámica del oleaje y los aportes fluviales en la conexión, transferencia, exportación e importación de agua, sustancias y calor como factor de transporte de vida y de contaminantes. Las características y eficiencia de este sistema de intercambio de flujos es el principal factor determinante de la biodiversidad de la Bahía.

Geográficamente se encuentra dividida en tres partes: (1) la bahía exterior, (2) la bahía interior y el (3) saco central. Esta geometría natural ha visto acentuada su irregularidad por medio de las diversas estructuras artificiales construidas por el hombre, como son los distintos diques del Puerto de Cádiz o el Puente de Carranza, y en el futuro con el dragado para la ampliación del Puerto de Cádiz y el Puente de "La Pepa" (Fig. 1).

La Bahía Externa (Fig. 1), situada al Norte del Estrecho de Puntales, conecta directamente al Océano Atlántico. El Sector Central (Fig 1) comprende el Estrecho de Puntales. Esta franja conecta el saco externo con el interno, y en ella se concentran la mayoría de las infraestructuras portuarias y de transportes. Además se están construyendo la ampliación del puerto y el nuevo puente. La Bahía Interna (Fig. 1), situada al sur y sureste del Estrecho de Puntales, baña las costas de San Fernando y Puerto Real y conecta con las Marismas y el Caño de Sancti-Petri.
La Bahía Externa se caracterizada por tener profundidades medias de $7 \mathrm{~m}$ con pendientes del $8 \%$ aproximadamente. En el sector Central las profundidades y pendientes son mayores debido a la presencia del canal, ya que llegan a pasar de $5 \mathrm{~m}$ a 20 $m$ en poca distancia habiendo un mayor calado para la navegación. Sin embargo, conforme se avanza hacia el Saco Interno, se observa como la batimetría va suavizándose, teniendo profundidades máximas de $6 \mathrm{~m}$ en la dirección del Caño de Carraca y de 2-3 $\mathrm{m}$ para la parte restante.

El sustrato está compuesto por materiales gruesos (arenas, con diámetros medios entre 2 y 0,02 $\mathrm{mm}$ ), suministrados básicamente por el mar, y materiales finos (limos y arcillas, con diámetros medios menores a $0,02 \mathrm{~mm}$ ) que son aportados por los ríos y que son depositados en cada marea en las zonas de baja energía, al confluir aguas dulces con un gran contenido de partículas en suspensión y aguas marinas, de alta salinidad.

\subsection{Forzamientos}

La dinámica y el transporte sedimentario que se genera en ésta área se ve afectado por los forzamientos procedentes del Océano Atlántico y del Golfo de Cádiz.

Las temperaturas de la zona se caracterizan por unos valores medios anuales relativamente altos $\left(16,7^{\circ}\right)$ con una moderada variación estacional. Respecto a las precipitaciones el rasgo más característico es su relativa escasez e irregularidad. La precipitación total anual registrada sobrepasa sensiblemente los $600 \mathrm{~mm}$.

El análisis realizado sobre los datos del WANA 1052046 (Fig. 1), facilitado por Puertos del Estado (Ministerio de Fomento), caracteriza el oleaje $\mathrm{y}$ viento en profundidades indefinidas. El conjunto de datos de este punto contienen los estimaciones diarias de oleaje (modelo WAM) y viento (modelo HIRLAM) desde 1995. Esta información se ha usado para analizar el clima marítimo y seleccionar las condiciones de forzamiento predominantes en la zona de estudio.

La direccionalidad del oleaje se puede resumir como W (32.7\%), WNW (27.7\%), WSW (7.88\%), $\mathrm{SE}(7.14 \%)$ y $\operatorname{ESE}(5.69 \%)$ (Fig. 2). La condiciones energéticas medias $\left(1.5 \mathrm{~m} \leq H_{s}\right)$ son las que predominan $(63.18 \%)$, mientras que las condiciones energéticas moderadas $\left(1.5 \mathrm{~m} \leq H_{s} \leq 3 \mathrm{~m}\right)$ y las 
WANA 1052046

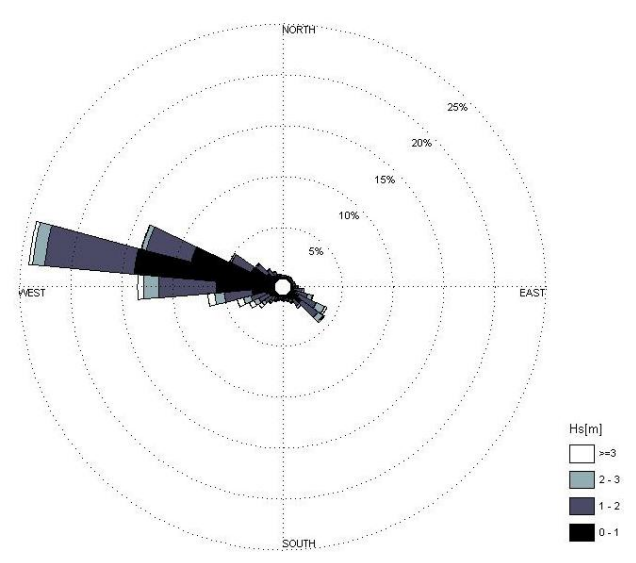

Figura 2: Rosa de oleaje

extremas $\left(H_{s} \geq 3 \mathrm{~m}\right)$ son menos frecuentes $(32.7 \%$ y $4.32 \%$, respectivamente). Los periodos de oleaje son generalmente altos ( $\leq 15 \mathrm{~s})$ llegando a alcanzar en excepciones los 21s. Los vientos predominantes proceden de W (10.7\%), WNW (10.6\%), E (10\%) y NW $(9.67 \%)$. Los vientos máximos registrados son de $22.6 \mathrm{~m} / \mathrm{s}$, siendo los valores medios de $13.68 \mathrm{~m} / \mathrm{s}$. La marea astronómica semidiurna presenta máximas variaciones de entre 2 a $3.5 \mathrm{~m}$.

\section{Metodología}

El objetivo de este trabajo, enmarcado en un proyecto de excelencia de la Junta de Andalucía, es (1) realizar una campaña de campo de larga duración para caracterizar la hidrodinámica de la Bahía y (2) aplicar, calibrar y validar un modelo numérico hidrodinámico que permita simular distintos escenarios geométricos o de forzamiento en la Bahía. La metodología de trabajo planteado se basa en los aspectos anteriores.

\subsection{Campaña de campo}

Entre Diciembre de 2011 y Mayo de 2012, se realizó una campaña en la Bahía de Cádiz y su entorno empleando un total de 13 instrumentos (Fig. 3). Se instalaron 5 correntímetros distanciados 5,5 km desde el primero, en la Bahía Externa, hasta el último, en el Saco interno. En algunos puntos de medida se ubicaron también sensores de conductividad y turbidez. Finalmente, se instalaron tres mareógrafos en puntos estratégicos dentro de la Bahía, como son los Caños y el Río San Pedro.
La posición del instrumento de la Boya Reviro (BR), a una profundidad de $10 \mathrm{~m}$, se seleccionó por la proximidad a la zona de ampliación del Puerto de Cádiz. Los tres siguientes instrumentos se colocaron en la Boya Obra (BO), Boya F (BF) y Boya Zona Franca N ${ }^{\circ} 2$ (BZF), con profundidades de 11 $\mathrm{m}, 13 \mathrm{~m}$ y $8 \mathrm{~m}$ respectivamente. Se ubicaron en esas posiciones motivado por (1) la construcción del nuevo puente "La Pepa" y (2) por los intercambios de aguas entre la parte Externa e Interna. Por último, para poder caracterizar el Saco interno se colocó un último punto de observación pasado el Puente de Carranza en la Boya Poste Luz (BP) (a una profundidad de 6m) (Fig 3). Los periodos de muestreo de cada instrumento se muestran en la tabla 1.

En BR se fondearon un ADCP de $2 \mathrm{MHz}$ en posición horizontal junto a un CT. El único problema que se presentó fue el agotamiento de la batería del ADCP, dejando de medir desde el 15 de Marzo (Fig. 3).En BO se ubicaron un AWAC de $1 \mathrm{MHz}$, un CT y un OBS-3+. En este caso por motivos desconocidos el instrumento estuvo un periodo de tiempo sin medir. Se han analizado los datos existentes, y en un análisis preliminar se ha creído conveniente sólo utilizar los correspondientes entre las fechas 15 de Marzo al 18 de Abril (Fig. 3). En BF se colocaron un ADCP de $1 \mathrm{MHz}$ en posición vertical junto a un CT y OBS-3+. Del 7 al 16 de Febrero no se tienen datos por que se agotó la batería (Fig. 3). En $\mathrm{BZF}$ se fondearon un $\mathrm{ADCP}$ de $2 \mathrm{MHz}$ en posición vertical y en $\mathrm{BP}$ un $\mathrm{ADCP}$ de $2 \mathrm{MHz}$ en posición horizontal. En éste último no se registró ningún problema. Sin embargo para el primero se ha realizado un tratamiento de los datos para eliminar los valores erróneos e inadecuados (Fig. 3). Por último los mareógrafos se ubicaron en el Río San Pedro, la zona de la Gallinera y de San Vicente. Son sitios estratégicos por sus descargas hacia la Bahía. Tuvieron un rango de muestreo de $1 \mathrm{~Hz}$ cada 900 segundos (Fig. 3). La programación en el caso de los OBS-3+ es según el módulo mareal de los ADCP y AWAC, cada 900 segundos un dato. Los CT muestrean con una frecuencia de $1 \mathrm{~Hz}$ cada 15 minutos.

Los ADCP estuvieron en una posición media de $1,5 \mathrm{~km}$ del margen derecho de la Bahía hacia el interior (Fig. 3), montados verticalmente a unos 0,55 $\mathrm{m}$ por encima del fondo del mar en un muerto de hormigón. El sensor muestrea velocidades orbitales, temperaturas y presiones con un rango de muestreo de $1 \mathrm{~Hz}$ cada 900 segundos, dando su promedio ca- 
da 120 segundos. En el caso del muestreo asociado al oleaje, se obtenían valores con una frecuencia de $1 \mathrm{~Hz}$ por cada hora durante 1024 segundos. En el caso del AWAC la ubicación y configuración fue similar a los ADCP, con una única diferencia en los resultados, ya que éste instrumento mide la superficie libre.

La hidrodinámica en profundidades indefinidas y las condiciones de viento durante la campaña de campo fueron controladas por la Boya Golfo de Cádiz y el punto WANA 1052046 (datos facilitados por Puertos del Estado, España), ubicados a 450 metros de profundidad y a 100 kilómetros al Oeste de Cádiz. Principalmente se van a utilizar los datos de la Boya ya que son medidas procedentes de la Red de Boyas de Aguas Profundas, que proporcionan observaciones reales de grandes zonas litorales generando internamente un conjunto de parámetros estadísticos que caracterizan cada uno de los agentes observados (Fig. 1).

Se ha realizado un estudio preliminar exhaustivo de los datos observados, ya que existían valores erróneos e inusuales. Primero se realizó una filtración de los datos en bruto eliminando las anomalías, para realizar unos ficheros que solo tuviesen los valores fiables. Luego, a través de análisis numéricos y estadísticos se han calculado las distintas variables. Con la presión se ha realizado un enfoque no lineal para un grupo de olas irregulares a través de la formulación de Nielsen (Nielsen, 1986), obteniendo así la superficie libre. Todos los cálculos fueron validados con los datos exportados del Storm, programa específico de la instrumentación.

\subsection{Modelo numérico}

El módulo principal del modelo DELFT3D realiza los cálculos hidrodinámicos, simultáneamente con los del transporte de la salinidad y el calor (módulo FLOW). La ventaja principal de este módulo es que permite estudiar: (1) los procesos tridimensionales, (2) los efectos de la densidad de sedimento en suspensión (que puede producir corrientes y/o amortiguación de la turbulencia) al estar incluidos en los cálculos hidrodinámicos (3) y los cambios en la batimetría, que puede ser incorporados a los cálculos hidrodinámicos (Lesser et al., 2004).

El sistema de ecuaciones tiene en cuenta la ecuación de cantidad de movimiento horizontal, la ecuación de continuidad, la ecuación del transporte y el modelo de cierre turbulento. Se prescinde de la ecuación de conservación de cantidad de movimiento vertical, ya que en la relación entre la presión hidrostática con las aceleraciones verticales, las aceleraciones verticales se presuponen más pequeñas que la aceleración gravitacional y por tanto no se considera. Esto hace que el modelo DELFT3D-FLOW sea adecuado para predecir el comportamiento mareal en profundidades reducidas. Uno de sus requisitos es que las escalas de longitud horizontal y del tiempo sean significativamente mayores que las escalas verticales. El modelo tiene en cuenta los gradientes de densidad horizontales que pueden ser causados por la salinidad y por los gradientes de temperatura a lo largo de la zona de estudio (Lesser et al., 2004). Se debe elegir si resolver las ecuaciones de hidrodinámica en coordenadas Cartesianas, Curvilíneas Ortogonales o Esféricas. En las simulaciones en tres dimensiones se suelen utilizar las Cartesianas.

\subsection{Ecuaciones del modelo}

El módulo DELFT3D-FLOW, basado en el desarrollo de la hidrodinámica para sistemas costeros y estuarios, resuelve las ecuaciones completas de Navier-Stokes y transporte utilizando la aproximación de Boussinesq en aguas someras (Lesser et al., 2004; Carballo, 2009; Iglesias and Carballo, 2009; Iglesias et al., 2012). Las ecuaciones se resuelven mediante un esquema en diferencias finitas de tres dimensiones.Las ecuaciones del modelo son:

$$
\frac{\partial u}{\partial x}+\frac{\partial v}{\partial y}+\frac{\partial w}{\partial z}=Q
$$

$$
\begin{aligned}
\frac{D u}{D t} & =f v-g \frac{\partial \zeta}{\partial x}-\frac{g}{\rho_{0}} \int_{z^{\prime}=z}^{z^{\prime}=\varsigma} \frac{\partial \rho}{\partial x} d z^{\prime}+ \\
& +v_{h}\left(\frac{\partial^{2} u}{\partial^{2} x}+\frac{\partial^{2} u}{\partial^{2} y}\right)+v_{v}\left(\frac{\partial^{2} u}{\partial^{2} z}\right) \\
\frac{D v}{D t} & =f u-g \frac{\partial \zeta}{\partial y}-\frac{g}{\rho_{0}} \int_{z^{\prime}=z}^{z^{\prime}=\varsigma} \frac{\partial \rho}{\partial y} d z^{\prime}+ \\
& +v_{h}\left(\frac{\partial^{2} v}{\partial^{2} x}+\frac{\partial^{2} v}{\partial^{2} y}\right)+v_{v}\left(\frac{\partial^{2} v}{\partial^{2} z}\right)
\end{aligned}
$$

$$
\frac{\partial \rho}{\partial z}=-\rho g
$$




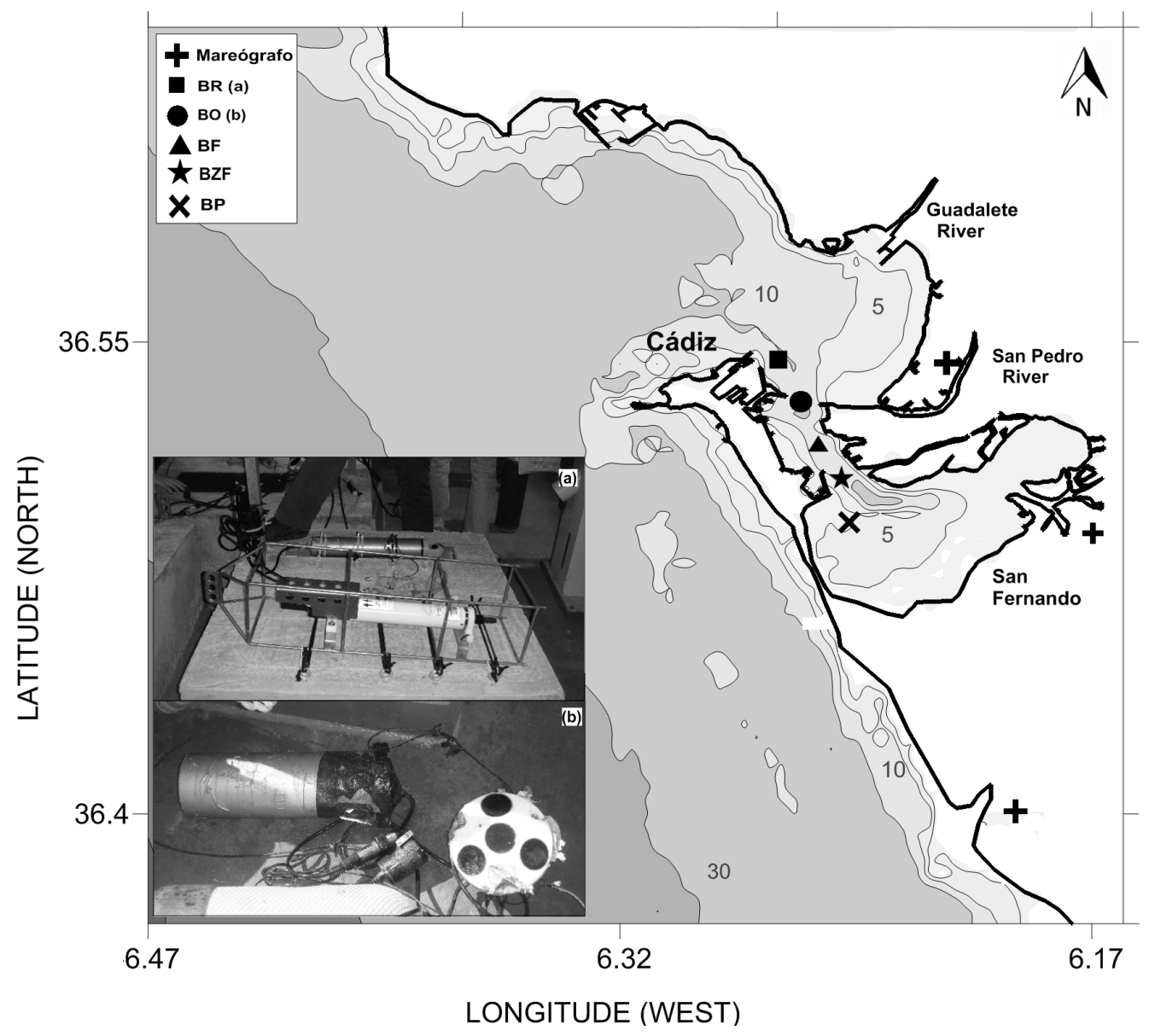

Figura 3: Ubicación de los instrumentos

\begin{tabular}{|c|c|c|c|}
\hline Instrumento & Localización & Fecha de fondeo & Fecha de datos \\
\hline $\mathrm{CT}$ & \multirow{2}{*}{ BR } & \multicolumn{2}{|c|}{$22 / 12 / 11-18 / 04 / 12$} \\
\hline ADCP & & $22 / 12 / 11-18 / 04 / 12$ & $22 / 12 / 11-15 / 03 / 12$ \\
\hline $\mathrm{CT}$ & \multirow{2}{*}{ BO } & \multicolumn{2}{|c|}{$22 / 12 / 11-18 / 04 / 12$} \\
\hline AWAC OBS & & $22 / 12 / 11-18 / 04 / 12$ & $15 / 03 / 12-18 / 04 / 12$ \\
\hline CT & \multirow[b]{2}{*}{$\mathrm{BF}$} & \multicolumn{2}{|c|}{$22 / 12 / 11-18 / 04 / 12$} \\
\hline ADCP OBS & & $22 / 12 / 11-18 / 04 / 12$ & $22 / 12 / 11-18 / 04 / 12$ \\
\hline ADCP & BZF & \multicolumn{2}{|c|}{$22 / 12 / 11-18 / 04 / 12$} \\
\hline $\mathrm{ADCP}$ & $\mathrm{BP}$ & \multicolumn{2}{|c|}{$22 / 12 / 11-18 / 04 / 12$} \\
\hline \multirow{3}{*}{ Mareógrafos } & RSP & \multirow{3}{*}{\multicolumn{2}{|c|}{$10 / 02 / 12-22 / 05 / 12$}} \\
\hline & $\mathrm{G}$ & & \\
\hline & SV & & \\
\hline
\end{tabular}

Cuadro 1: Periodos 


$$
\frac{D c}{D t}=D_{h}\left(\frac{\partial^{2} c}{\partial^{2} x}+\frac{\partial^{2} c}{\partial^{2} y}\right)+D_{v} \frac{\partial^{2} c}{\partial^{2} z}-\lambda_{d} c+R_{S}
$$

La ecuación (1) expresa la conservación de la masa bajo la hipótesis de incomprensibilidad; las ecuaciones (2) representan la conservación de cantidad de movimiento en direcciones $x$ - e $y$ - respectivamente; la ecuación (3) expresa la conservación de cantidad de movimiento en dirección $z$ - (dirección vertical), en la que se ha simplificado la presión hidrostática asumiendo aguas someras reducidas; la ecuación (4) es la ecuación de transporte, la cual resuelve tanto la salinidad como la temperatura. En las ecuaciones, $\zeta$ es el nivel del mar referenciada al plano $z=0 ; u, v$ y $w$ son las componentes de las velocidades en dirección $x$-, $y$ - y $z$ - respectivamente; $Q$ representa el aporte de una fuente externa por unidad de área; $f$ es el parámetro de Coriolis, $v_{h}$ y $v_{v}$ son los coeficientes de viscosidad horizontal y vertical respectivamente; $\rho$ y $\rho_{0}$ son la densidad y la densidad referenciada a la del agua del mar respectivamente; $c$ son las variables temperatura o salinidad; $D_{h}$ y $D_{v}$ son los coeficientes de difusión horizontal y vertical respectivamente; $\lambda_{d}$ representa el proceso de demora de primer orden; y $R_{s}$ es la fuente térmica por unidad de área.

\subsubsection{Aplicación del modelo}

La batimetría de la Bahía de Cádiz ha sido generada a través de la unión de distintas batimetrías detalladas de la zona (Autoridad Portuaria de Cádiz). A ésta se le unió la topografía de las zonas colindantes extraídas del Modelo Digital del Terreno (Junta de Andalucía) con una resolución de 10x10m. El modelo hidrodinámico fue inicializado con una malla en coordenadas cartesianas que cubría la Bahía de Cádiz y su entorno. El tamaño de la malla es de $245 \times 245$ celdas, suficiente para capturar las profundidades relevantes y características de la Bahía.

Las condiciones iniciales del modelo numérico, superficie libre, salinidad y temperatura han sido los valores en el instante inicial de los instrumentos de BR. Respecto al problema de cierre turbulento se ha usado el modelo k- $\epsilon$ (Rodi, 1984). Las condiciones de contorno asociadas al terreno han sido nulas. Sin embargo en el contorno "Oeste" $(2,1 ; 244,1)$ se han impuesto condiciones abiertas del tipo de nivel con forzamiento astronómico, para lo que se han

\begin{tabular}{|c|c|c|}
\hline Armónico & Amplitud (m) & Fase $\left(^{\circ}\right.$ ) \\
\hline M2 & 1.0246 & 57.63 \\
\hline S2 & 0.3702 & 83.907 \\
\hline SA & 0.0614 & 229.87 \\
\hline Q1 & 0.0227 & 251.15 \\
\hline O1 & 0.067 & 302.79 \\
\hline P1 & 0.0213 & 35.55 \\
\hline K1 & 0.0671 & 42.66 \\
\hline 2N2 & 0.0344 & 31.75 \\
\hline MU2 & 0.0389 & 18.44 \\
\hline N2 & 0.2167 & 41.41 \\
\hline NU2 & 0.0401 & 46.9 \\
\hline L2 & 0.0199 & 74.02 \\
\hline T2 & 0.0215 & 84.16 \\
\hline R2 & 0.006 & 68.41 \\
\hline K2 & 0.1058 & 80.41 \\
\hline MN4 & 0.0126 & 117.71 \\
\hline M4 & 0.0282 & 160.34 \\
\hline MS4 & 0.013 & 229.81 \\
\hline
\end{tabular}

Cuadro 2: Armónicos de marea (Río San Pedro)

tenido que obtener los armónicos de marea, seleccionando los datos del mareógrafo situado en el Río San Pedro por ser el más externo a la Bahía. (Tabla 2). Para las condiciones de transporte se han definido previamente tres capas $(40 \%, 50 \%, 10 \%)$ eligiendo un perfil vertical lineal, donde en el fondo se definirán como valores los correspondientes a BR y en superficie los valores pertenecientes a la Boya de Cádiz. De la Boya Golfo de Cádiz se ha podido obtener la velocidad y dirección del viento necesarias para su aplicación en el modelo.

Tras definir los parámetros fijos, a continuación se calibran los principales parámetros del modelo: el coeficiente de arrastre por viento, la rugosidad y la viscosidad. Para un primer tanteo se usaran los datos utilizados en estudios anteriores (Álvarez et al., 2003). El periodo de simulación será un intervalo donde no exista ningún tipo de forzamiento, siendo el único agente la marea.

La coherencia entre el modelo y lo observado es analizada por el error medio cuadrático (RMSE), el coeficiente de correlación (R), y el coeficiente de Skill (S). Considerando que $M_{n}$ y $C_{n}$ son los datos observados y los modelados, respectivamente, a $\mathrm{N}$ puntos.

El RMSE viene dado por: 


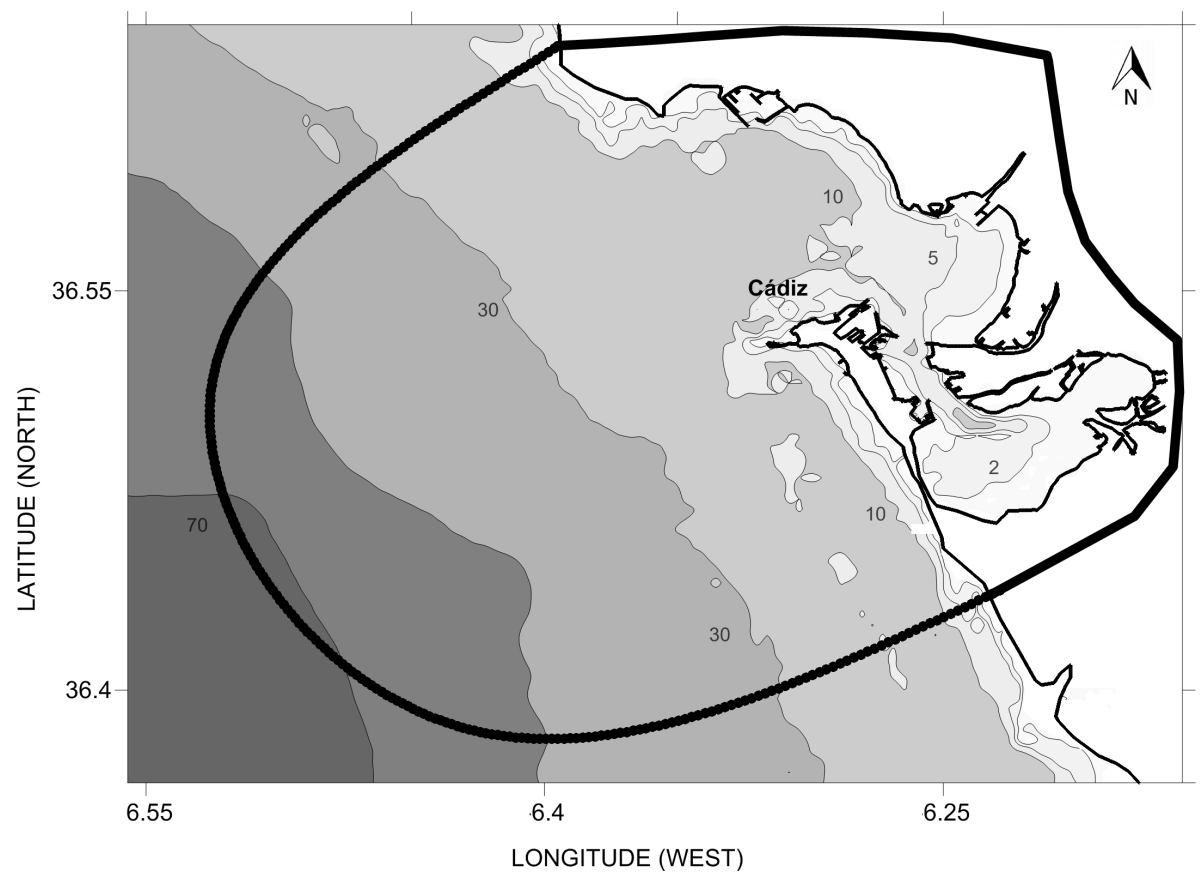

Figura 4: Malla del modelo DELFT-3D

$$
R M S E=\left[\frac{1}{N} \sum_{n=1}^{N}\left(M_{n}-C_{n}\right)^{2}\right]^{\frac{1}{2}}
$$

El coeficiente de correlación $(R)$ entre $M_{n}$ y $C_{n}$ es definido por:

$$
R=\frac{\frac{1}{N} \sum_{n=1}^{N}\left(M_{n}-\bar{M}_{n}\right)\left(C_{n}-\bar{C}_{n}\right)}{\sigma_{C} \sigma_{M}}
$$

donde $\sigma_{M}$ y $\sigma_{C}$ son las desviaciones típicas de los datos observados y modelados, respectivamente. La barra superior significa la media de la serie de datos. Los rangos de correlación van desde 0 (mala correlación) hasta 1 (buena correlación).

El coeficiente de Skill $(S)$ propuesto por Wilmott [1981] viene dado por:

$$
S=1-\frac{\sum_{n=1}^{N}\left|C_{n}-M_{n}\right|^{2}}{\sum_{n=1}^{N}\left(\left|C_{n}-\bar{M}_{n}\right|^{2}+\left|M_{n}-\bar{M}_{n}\right|^{2}\right)}
$$

donde los rangos del coeficiente de Skill van desde 0 (mala correlación) hasta 1 (buena correlación).

\section{Resultados}

\subsection{Descripción de la campaña}

La campaña de campo se realizó entre el 22 de Diciembre del 2011 y el 18 de Abril de 2012. Durante ese periodo la Bahía de Cádiz estuvo sometida a los forzamientos que se representan en la figura 5 , donde la línea negra corresponde a los datos medidos por la Boya de Cádiz, la gris a los datos del punto WANA 1052046 y los puntos al instrumento ubicado en BR. Se observa que los valores medios de las alturas de ola significantes fueron del orden de $1.5 \mathrm{~m}$, con periodos de pico de 10s. Las carreras de marea para el caso de mareas vivas fueron de 3.5-4 m, mientras que en mareas muertas se redujeron a casi la mitad (1.5-2 m). Predominaron los vientos del SE-SW con velocidades de 6-7 m/s.

Si se analizan periodos de tormenta, a finales de Marzo del 2012, los valores de altura de ola son más del doble que de los valores medios $(3.5 \mathrm{~m})$, sin embargo el periodo de pico es menor (6 $\mathrm{s})$. Los vientos predominantes son los procedentes del SSW y SSE, con velocidades de hasta $12 \mathrm{~m} / \mathrm{s}$. Los periodos de pico máximos se alcanzan cuando los vientos proceden de SSW y SW con valores inferiores a los 10s. En estos periodos las alturas de ola toman valores de alrededor de $1 \mathrm{~m}$.Sin embargo, esta tormenta no coincidió con valores máximos de superficie libre. 
Comparando los resultados del instrumento ubicado en el saco externo con los de la Boya, puede observarse que las alturas de ola máximas $(\mathrm{Hs} \approx 1.4 \mathrm{~m})$ se alcanzan con vientos procedentes del NE y N. Estos vientos suelen alcanzar velocidades de $\approx 7 \mathrm{~m} / \mathrm{s}$. Justo en estos periodos se dan los valores máximos de superficie libre tanto en mareas vivas como muertas. Sin embargo cuando se dan los vientos procedentes del $\mathrm{S}$ o próximos a éste, la altura de ola no se ve afectada por este agente (Fig. 5).

Las corrientes de marea siguen distintas direcciones dependiendo de la ubicación (Fig. 6). En la parte externa se tiene una única dirección, la del canal. En la zona superior del sector central se tienen dos direcciones: la del canal y la horizontal. Esta última coincide con la del instrumento ubicado en la parte inferior del canal. Llama la atención las direcciones del Sector Central. Esto puede explicarse debido a su cercanía al puerto situado en Zona Franca y al puente de Carranza, y por encontrarse en la zona de intercambio de flujos entre los sacos (Fig. 6). Aún así se analizará los valores de estos puntos por sus resultados extraños.

Las corrientes máximas son alcanzadas en BR, por su situación más expuesta y abierta. La desaceleración de las corrientes llega hasta BZF, donde los valores son mínimos. Sin embargo existe cierta aceleración en las corrientes en BP por la apertura del Estrecho de Puntales hacia el saco interno (Fig $6)$. Se dan concentraciones de las corrientes en BF y BZF. En el primer caso se observa en la parte central. Sin embargo en el segundo se concentra hacia el lado izquierdo, cerca al puerto de Zona Franca. Por último decir que para BR y BP no existen concentraciones, y siguen un comportamiento "lineal" hacia su dirección principal (Fig. 6).

Para la representación de BF se ha tenido que reconstruir la señal, ya que durante una semana no se obtuvieron datos. La reconstrucción se ha hecho a partir de los armónicos de marea con la herramienta t_tide (Pawlowicz et al., 2002).

En la figura 7 se analizan las distintas señales de los instrumentos: (a) BR, (b) BO, (c) BF, (d) BZ y (e) BPL. No existen grandes variaciones entre los distintos puntos, pero si se realiza un pequeño zoom puede observarse variaciones de $0.2 \mathrm{~m}$ de BR con respecto a $\mathrm{BZ}$, y de $0.1 \mathrm{~m}$ de $\mathrm{BPL}$ con respecto a BR. El comportamiento mareal entre BR y BF es muy similar existiendo diferencias de $\mathrm{mm}$.

\subsection{Análisis Mareal}

El estudio de la propagación y transformación de las ondas en el Bahía se realiza con un análisis armónico (Pawlowicz et al., 2002). Específicamente, el período de análisis abarca desde el 16 de Febrero de 2012 al 14 de Marzo de 2012.

La marea es semidiurna $\frac{\eta_{K 1}+\eta_{O 1}}{\eta_{M 2}+\eta_{S 2}} \approx 0.08$. Las constituyentes asociadas con este período (M2, S2 y N2) son efectivamente las más enérgicas en la Bahía (Fig. 8). Las mayores amplitudes (M2) de estas constituyentes se alcanzan en el Saco Interno, y las mínimas en el Saco externo, yendo de menos a más. S2 sigue una propagación paralela a M2 pero con valores menores. Sus fases tienen un leve crecimiento conforme se avanza por la Bahía, teniendo dos notables diferencias en el primer y último punto. El comportamiento de las constituyentes diurnas es similar a la de las componentes semi-diurnas pero con valores menores.

Las componentes cuasi diurnas son principalmente generadas en el Saco Interno, aunque el valor máximo se alcanza en el Río San Pedro (Fig. 8). El comportamiento de ambas constituyentes es muy similar. Estas componentes contribuyen significativamente a la asimetría de la marea, y al transporte y acumulación de sedimentos. Sus fases también tienen un crecimiento lineal conforme se avanza hacia el interior de la Bahía. Las componentes sexta diurnas se comportan de manera similar a las anteriores pero con menor variabilidad espacial. Las amplitudes de la constituyente quincenal van disminuyendo a lo largo de la Bahía. Las fases también van cambiando. Al inicio, aumenta hasta llegar a BZ donde disminuyen. Sin embargo si se considera el margen de error, estas variaciones no san tan significativas.

Los efectos no lineales cobran importancia en aguas someras, distorsionando el perfil de la onda. Los armónicos de marea generados a partir de M2 son los que provocan esta asimetría. Cuando la marea entra en la Bahía, la relación $\eta_{M 4} / \eta_{M 2}$ aumenta de 0.021 a 0.029. Sin embargo el valor máximo se da en la desembocadura del Río San Pedro (0.034). Por otra parte la diferencia entre fases, $\left|2 \theta_{M 2}-\theta_{M 4}\right|$ es menor de $180^{\circ}$ en toda la Bahía, lo que indica que en la Bahía domina la llenante según citetSpee1984. Para el estudio de las variaciones del nivel del mar y de las corrientes entre los ciclos de mareas vivas y muertas se ha escogido como fechas 22/02/2012 

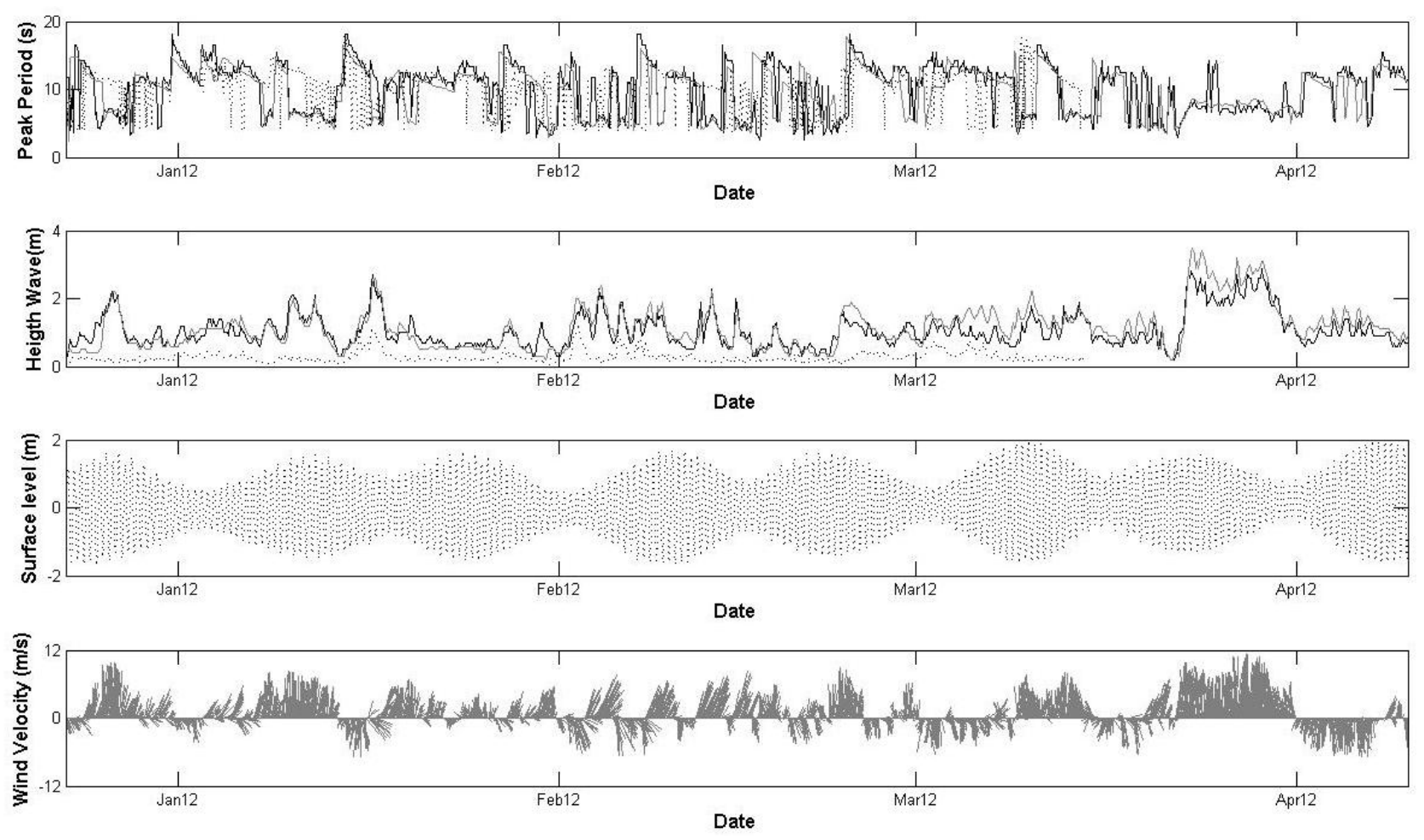

Figura 5: Tp, Hs, Nivel, Velocidad de Viento (Dirección de procedencia respecto al Norte)

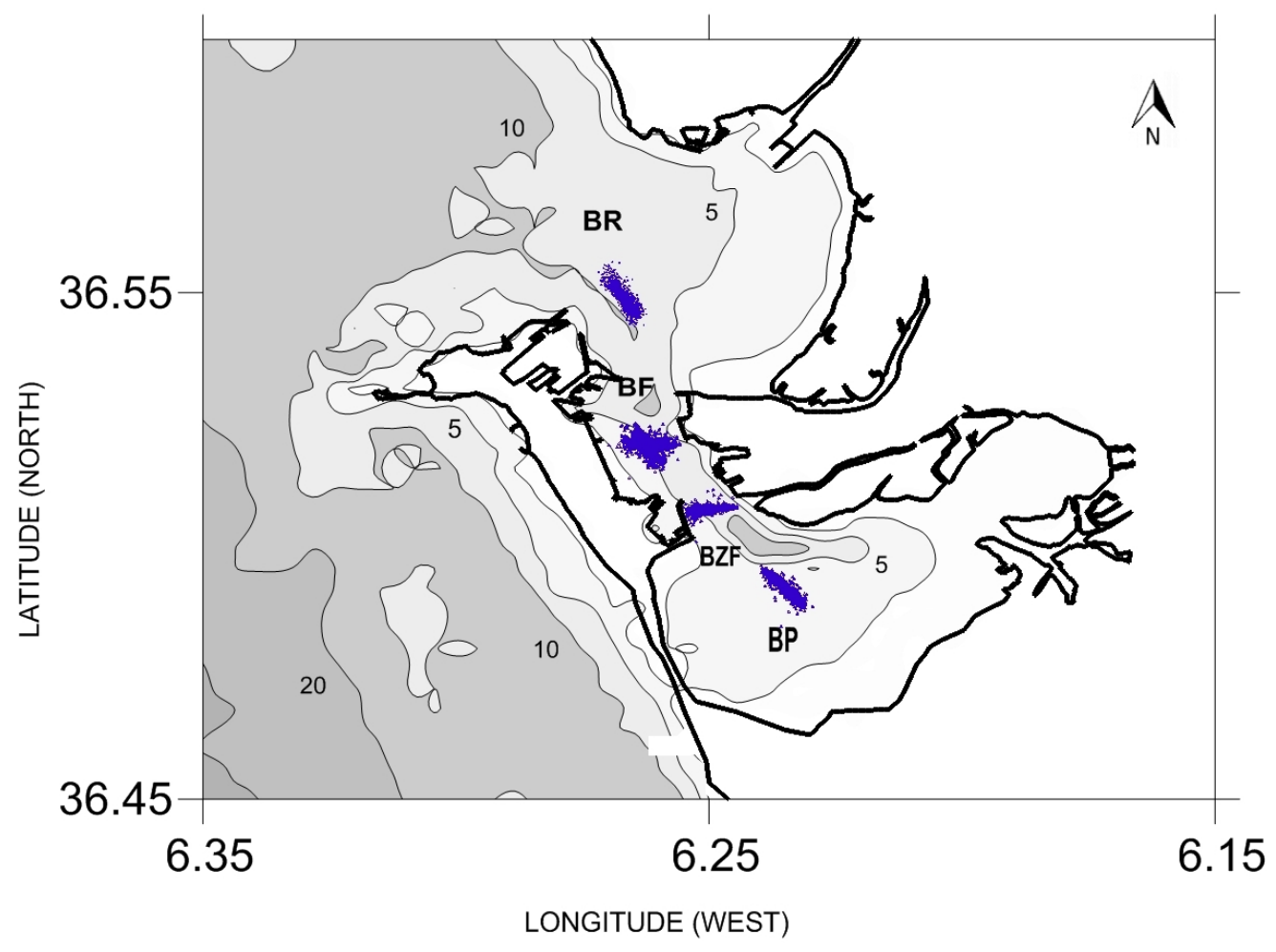

Figura 6: Rosas de Corriente 


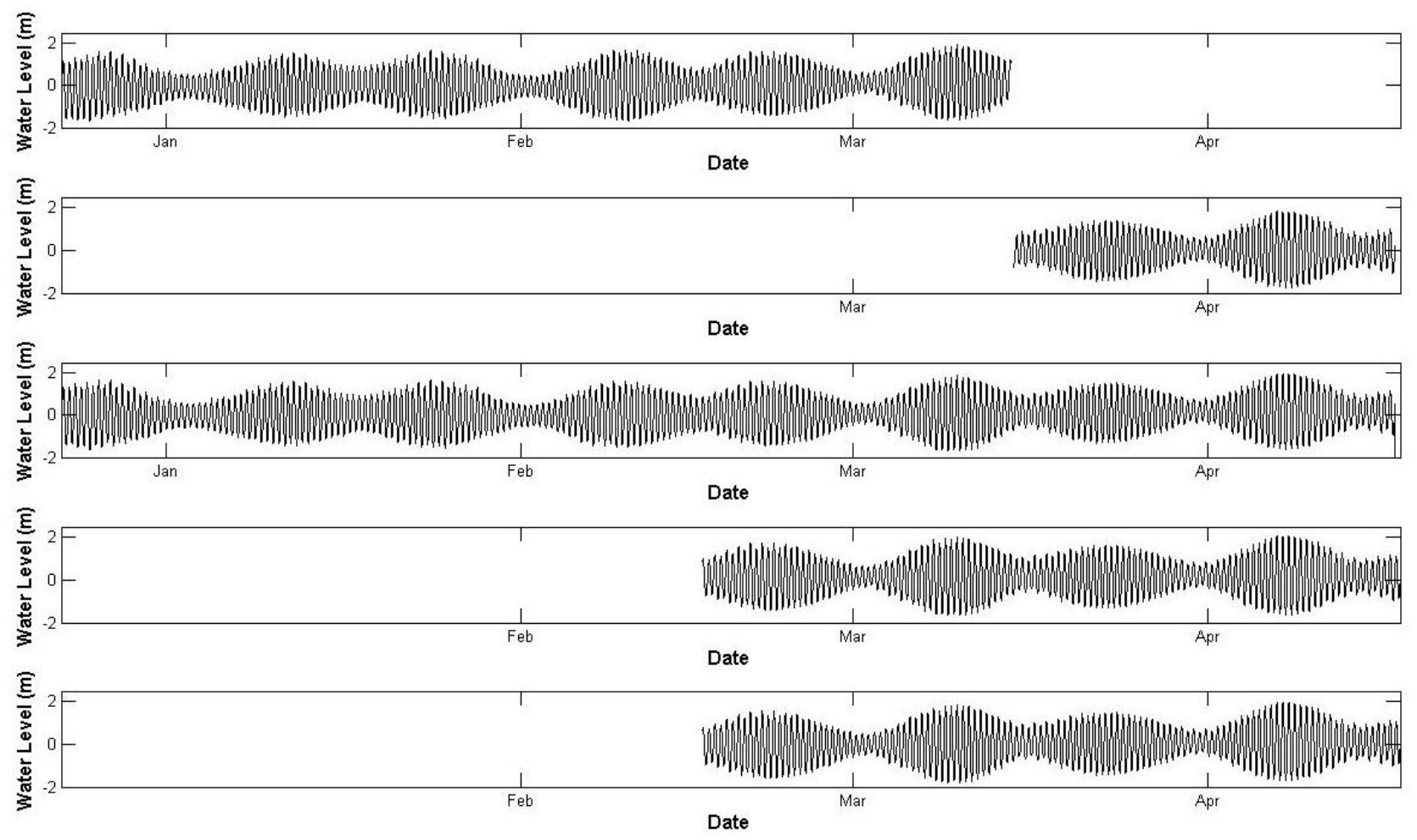

Figura 7: Nivel medio del mar en los diferentes intrumentos: (a) BR, (b) BO, (c) BF, (d) BZF y (e) BPL
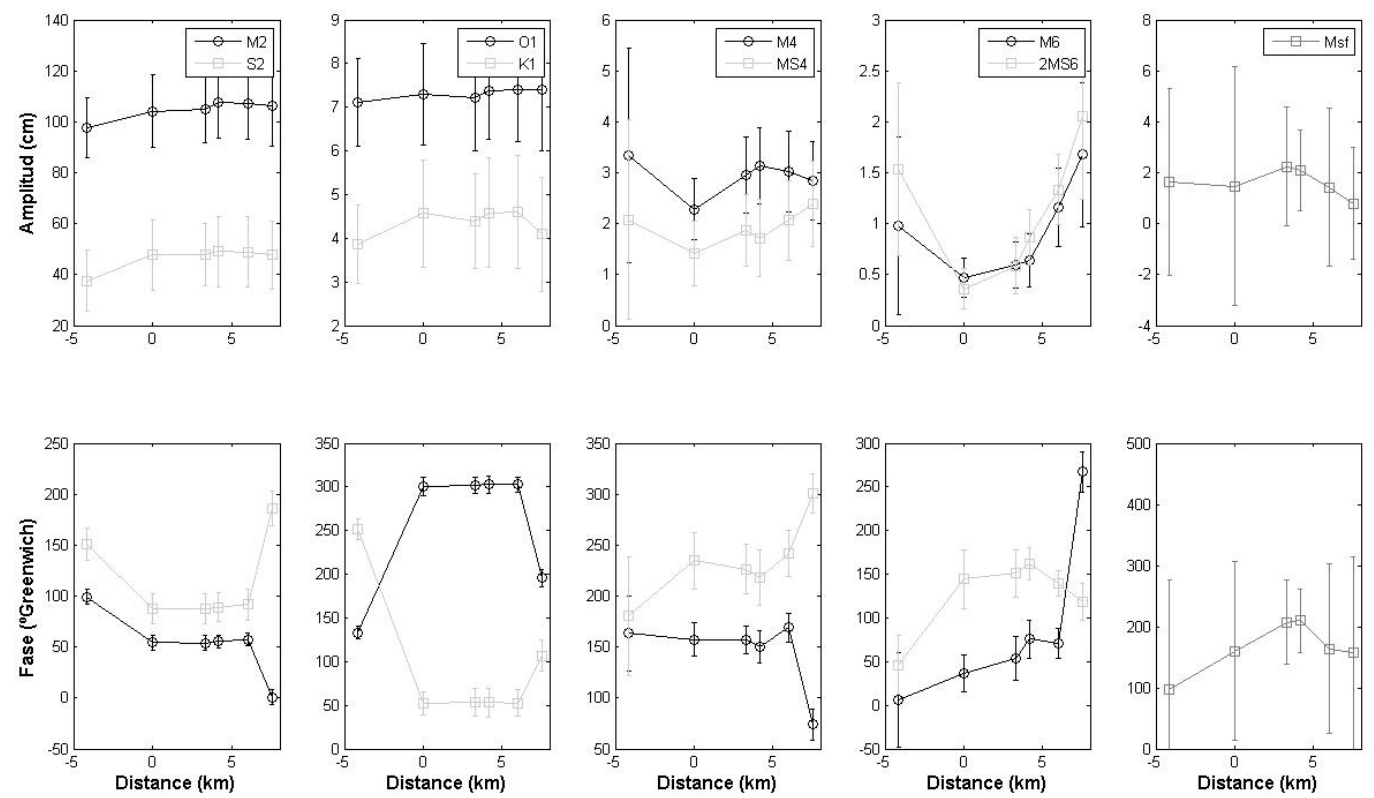

Figura 8: Análisis espacial armónico: (1) RSP, (2) BR, (3) BF, (4) BZ, (5) G 

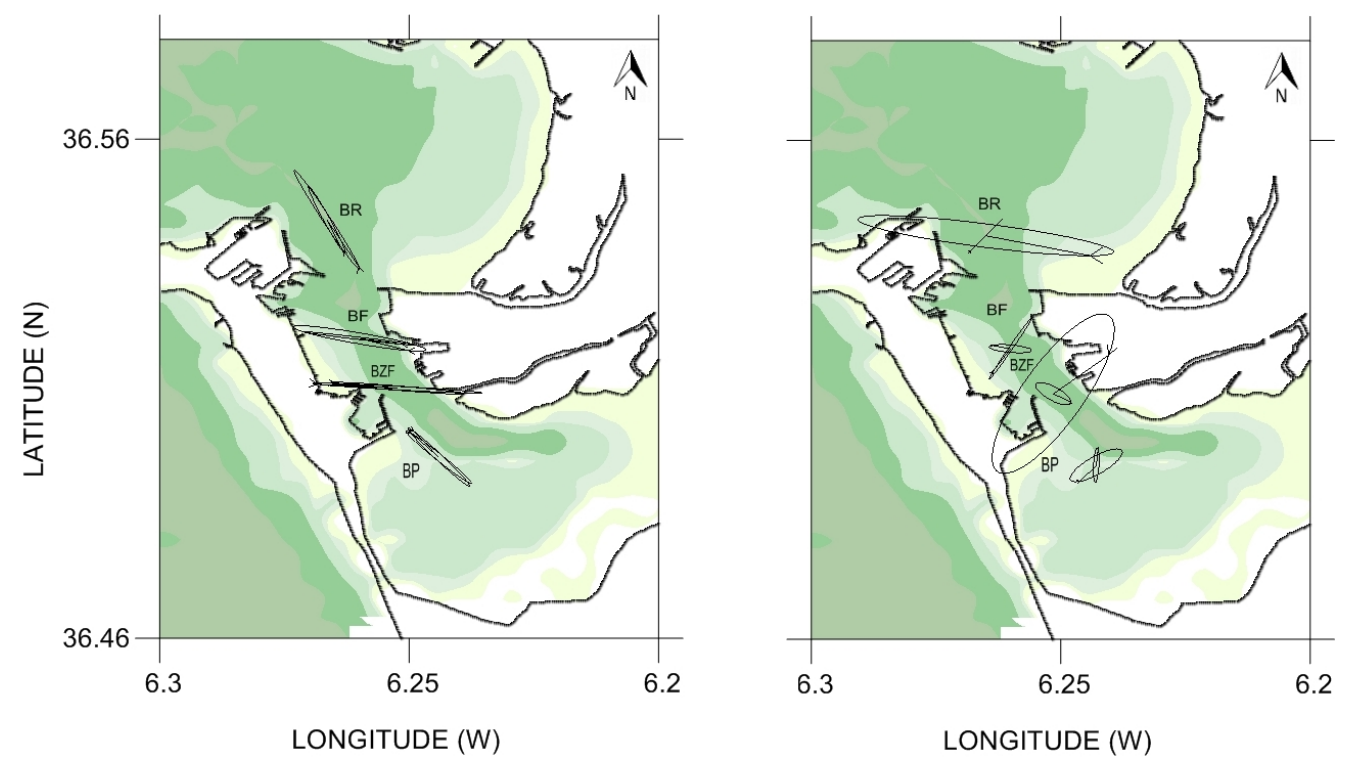

Figura 9: Elipses de marea: (a) Componente M2 y (b) Componente K1
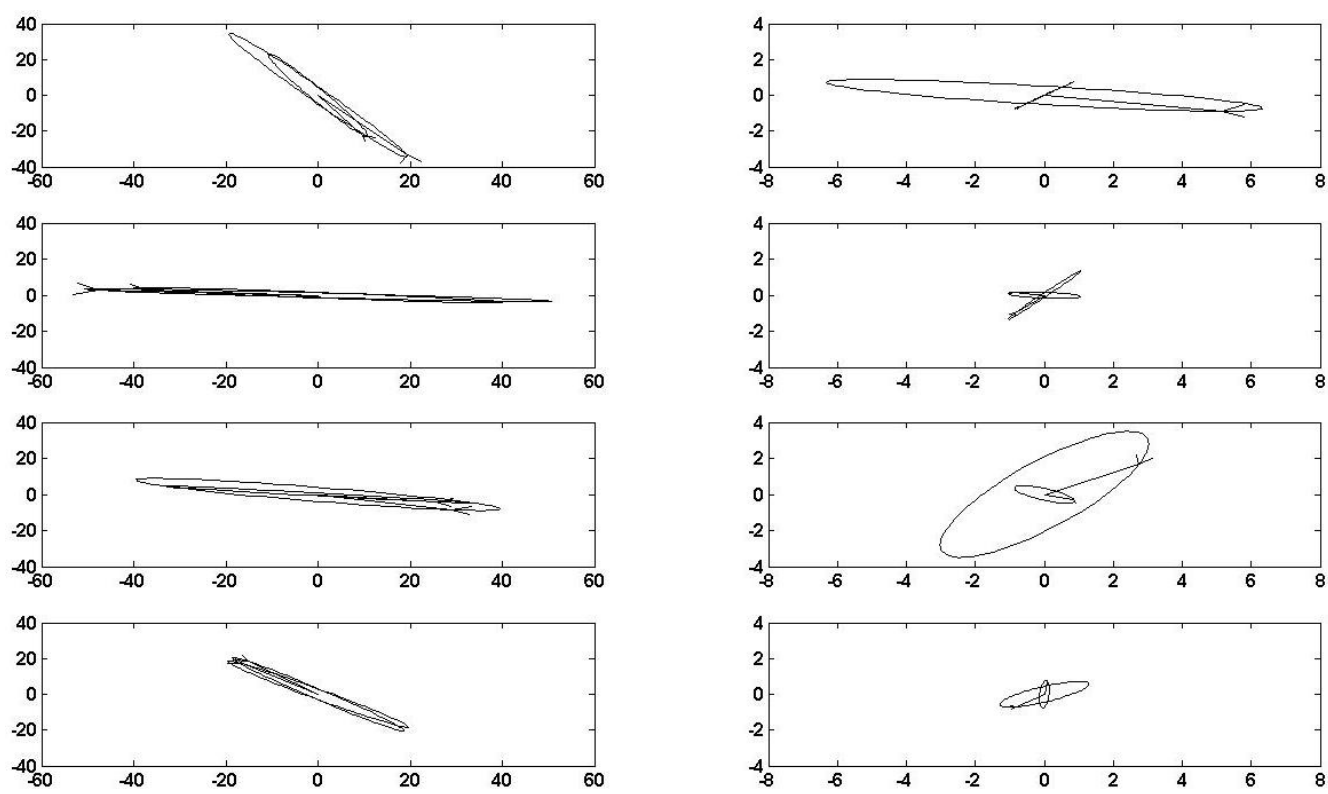

Figura 10: Elipses de marea: (a) Componente M2 (BR, BF, BZ, BPL) y (b) Componente K1 (BR, BF, BZ, BPL) 
y 01/03/2012 respectivamente. Todos los valores se han adimensionalizado respecto a BR.

En mareas muertas el nivel del mar va aumentando a lo largo del Canal de Puntales (1.10). Sin embargo al alcanzar la onda la Bahía Interna decrece (0.74). En cuanto a las mareas vivas, las amplitudes a lo largo de la Bahía toman valores semejantes al de referencia. Las oscilaciones de las velocidades a lo largo de la Bahía siguen un comportamiento similar entre mareas vivas y muertas, aunque las diferencias respecto al de referencia son mayores en el caso de las vivas. Las velocidades van aumentando a medida que la onda avanza por el canal hasta llegar al saco interno, donde la velocidad disminuye.

Con la diferencia entre las fases de $\eta$ y $u$ del armónico M2 se puede estudiar el movimiento oscilatorio en la Bahía de Cádiz. Los valores de esta diferencia están cercanos a $\pm \pi / 2$ por lo que se concluye que predominan las ondas estacionarias. En el caso de F y PL están cerca de $-\pi / 2$, esto quiere decir que existe un desfase en las corrientes con respecto a la superficie libre. Este comportamiento es contrario a $\mathrm{R}$ y $\mathrm{ZF}$.

Con el análisis armónico de las corrientes (Pawlowicz et al., 2002) se obtienen las elipses de marea para cada constituyente. En este caso solo se va a analizar las componentes M2 y K1, por ser las más representativas del comportamiento mareal (Figs. 9 y 10).

En M2 las direcciones de R y PL siguen la del canal, mientras que las de $\mathrm{F}$ y ZF siguen una dirección diferente. Este comportamiento en $\mathrm{F}$ y $\mathrm{ZF}$ se está estudiando, para ello se está analizando la velocidad en la dirección Up, por si existiese una circulación transversal significativa que provocase una dirección distinta a la del canal. Los errores en los ejes de las elipses de marea oscilan entre 2 y $7 \mathrm{~cm} / \mathrm{s}$, siendo los máximos errores en $\mathrm{BF}$, al igual que en los errores de fases se dan en este punto y BZ $\left( \pm 10^{\circ}\right)$. Estos errores hacen presuponer que los resultados son fiables.

Para la componente K1, los errores en los ejes varían entre 0.4 y $2.3 \mathrm{~cm} / \mathrm{s}$. Estos errores son excesivos teniendo en cuenta que los valores en K1 oscilan entre -4 y $4 \mathrm{~cm} / \mathrm{s}$. Al igual pasa con los errores en las fases que también alcanzan oscilaciones muy altas $\left(33^{\circ}-80^{\circ}\right)$. Estos máximos se dan en PL y Z. Por tanto los resultados obtenidos pueden distar mucho de la realidad.

\subsection{Calibración y validación del modelo}

\subsubsection{Flow}

Inicialmente sólo se va a calibrar la hidrodinámica de la Bahía inducida (principalmente) por la marea, para lo que se ha seleccionado un periodo de tiempo donde la influencia del viento y del oleaje sea despreciable. Las fechas seleccionadas fueron del 17/01/12 al 30/01/12 con intervalos de 15 minutos para coordinarlos con los instrumentos.

Se han seguido tres fases para garantizar la bondad de los resultados del modelo. Primero, se calibró el modelo con una propagación de la marea utilizando las 18 constituyentes mareales principales del mareógrafo de Tarifa y Bonanza. Segundo, el modelo ha sido evaluado comparándolo con los datos de la Boya y el WANA. Finalmente, se valida el modelo comparándolo con los resultados observados en las campañas realizadas.

\subsubsection{Calibración}

El modelado del prisma de marea y las corrientes a través de la Bahía de Cádiz requiere una representación correcta de la batimetría, así como un modelado preciso de las elevaciones y de la propagación de las mareas. La reconstrucción cuidadosa de los canales y características cercanas a la costa mejoró significativamente los resultados en este área. Las mediciones continuas e históricas de los niveles de agua y componentes armónicas de marea están disponibles en la Boya y WANA (Fig. 1). Una estimación inicial de las mareas se obtuvo forzando el modelo con las 12 principales constituyentes de marea de los mareógrafos situados en Bonanza y Tarifa. Estos resultados distaban mucho de la realidad por lo que se espero a tener los datos de la instrumentación ubicada en RSP (Fig. 3). Las presiones registradas en RSP fueron transformadas en superficie libre (Nielsen, 1986). Se realizó el análisis armónico de marea en los niveles de agua calculados durante los 4 meses de fondeo, utilizando la herramientas t_tide (Pawlowicz et al., 2002) (Tabla $2)$.

El modelo ha mostrado ser sensible a las variaciones en la batimetría y topografía, así como a la rugosidad y a los parámetros de calibración primaria. Los coeficientes de rugosidad de Chézy con los que se obtuvo el mejor ajuste fueron (80 (U), $60(\mathrm{~V}))$. Otro parámetro que ajustaba con mayor o menor exactitud el modelo es el coeficiente de arrastre de viento. Para una primera simulación se tomó 0.003 
(Álvarez et al., 2003) y se fue variando hasta llegar 0.005, que proporcionó el mejor ajuste.

\subsubsection{Validación}

Para validar el modelo hidrodinámico se han usado los datos de la campaña de campo. Se han comparado los resultados con los dos instrumentos que midieron en las mismas fechas (Tabla 1). La precisión de los valores modelados con respecto a los observados osciló entre 0.97 y 0.99 (Figs. 11 y 12). Sin embargo la precisión en las velocidades de corriente es menor (BF: 0.73-0.94; BR: 0.84-0.95) (Figs. 1316), aunque comparando con otros trabajos en los que se ha aplicado este modelo en entornos (Lesser et al., 2004; Carballo, 2009; Iglesias and Carballo, 2009; Iglesias et al., 2012; Elias and Hansen, 2012), se puede considerar un buen ajuste.

Una vez habiéndose validado el modelo, sólo queda corroborar su funcionalidad en otros intervalos de tiempo. Del 29 de Enero del 2012 al 10 de Febrero del 2012 (Figs. 11 - 16, puntos grises) se volvió a simular el modelo, con las mismas variables definidas en la calibración. Los resultados fueron muy similares a los observados. Se unieron ambas series temporales, y se obtuvo una correlación del nivel de $\mathrm{RMSE}=0.98$, y en el caso de las velocidades en dirección Este y Oeste, $\mathrm{RMSE}=0.89$ y RMSE $=0.94$ respectivamente.

\subsubsection{Dragado}

La necesidad del dragado $\left(3,2\right.$ millones de $\left.\mathrm{m}^{3}\right)$ se vio motivada por la construcción de una nueva terminal, en el dique de Levante, con una superficie de $22 \mathrm{Ha}$ y con un aumento del calado de $5.5 \mathrm{~m}$ sobre los $10,5 \mathrm{~m}$ con los que cuenta la zona actualmente. La figura 17 muestra las diferencias que existen entre la batimetría con (figura inferior) y sin (figure superior) dragado. Se ha realizado una variación en la batimetría del canal, desplazándola hacia la derecha para tener en cuenta la construcción de la nueva terminal. También se observa la diferencia que existe en las profundidades en el canal que aumentan a $16 \mathrm{~m}$ en la zona próxima a la terminal.

Se va a simular el modelo entre el 17 y el 30 de Enero del 2012, para así poder compararlos con los resultados anteriores. Dentro de este intervalo se han seleccionado cuatro instántes:las condiciones de máximo nivel (24/01/2012 03:45:00) y viento (20/01/2012 00:30:00) medidos durante la campaña, así como una situación de máxima velocidad de llenante (19/01/2012 22:15:00) y vaciante
(20/01/2012 04:30:00) (Fig. 18). Las máximas diferencias generadas, en el segundo y primer caso por orden, se concentran cerca del puerto de Cádiz y en la parte superior del Canal de Puntales. Sin embargo para la marea de llenante y de vaciante las variaciones son menores, dándose en la parte inferior y superior del Sector Central respectivamente. La Bahía Interna no experimenta grandes cambios.

\section{Conclusiones}

Esta tesina fin de máster se ha centrado en la caracterización hidrodinámica de la Bahía de Cádiz, situada al Sur-Oeste de la Península Ibérica, mediante medidas de campo y modelado numérico. Las medidas de campo se realizaron entre los meses de Diciembre de 2011 hasta Mayo de 2012 empleando un total de 13 instrumentos. El modelo hidrodinámico que se ha implementado y validado ha sido el DELFT3D (módulo Flow).

Las principales conclusiones son:

- Durante la campaña de campo predominaron las alturas de ola significantes de $1.5 \mathrm{~m}$ con periodos de $10 \mathrm{~s}$. En el mes de Marzo se produjo una tormenta con alturas de ola de hasta 3.5 $\mathrm{m}$. Las carreras de marea variaron de $4 \mathrm{~m}$ a 2 $\mathrm{m}$. Los vientos que predominan son los procedentes del SE-SW $(6-7 \mathrm{~m} / \mathrm{s})$.

- El nivel de marea tiene un comportamiento similar en toda la Bahía (con variaciones de $\pm 0,1$ $\mathrm{cm}$ ), aunque las componentes asimétricas aumentan conforme se avanza por la misma $( \pm 1$ $\mathrm{cm})$. En el comportamiento de las corrientes se observan distintas direcciones dependiendo de la ubicación en la Bahía. Tras el análisis de las fases entre $\eta$ y $u$ del armónico M2 se concluye que las ondas que predominan son las estacionarias $( \pm \pi / 2)$. Por otra parte la diferencia entre fases, $\left|2 \theta_{M 2}-\theta_{M 4}\right|$ es menor de $180^{\circ}$ en toda la Bahía, lo que indica que en la Bahía domina la llenante.

- Se ha implementado y aplicado el modelo hidrodinámico DELFT3D (módulo Flow). Dicho modelo se calibró comparando con los datos de campo medidos del 17/01/12 al 30/01/12, periodo durante el cual la influencia del viento y del oleaje fueron despreciables. El modelo ha mostrado ser sensible a las variaciones en 


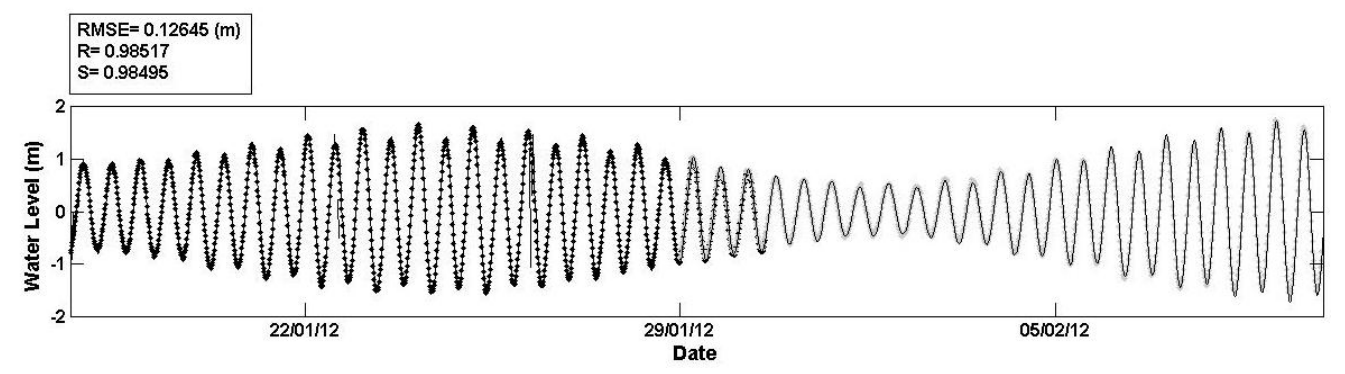

Figura 11: Nivel del mar (BR): Linea-Modelado y Puntos-Observado

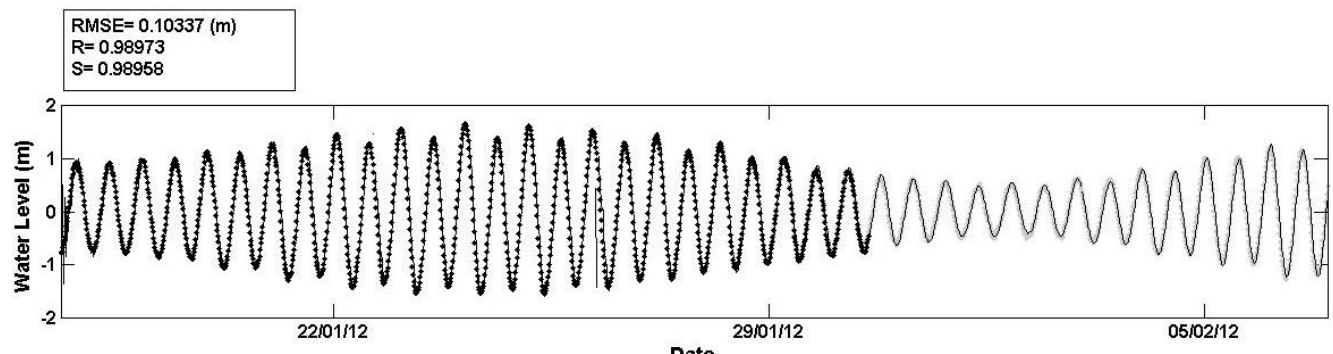

Figura 12: Nivel del mar (BF): Linea-Modelado y Puntos-Observado

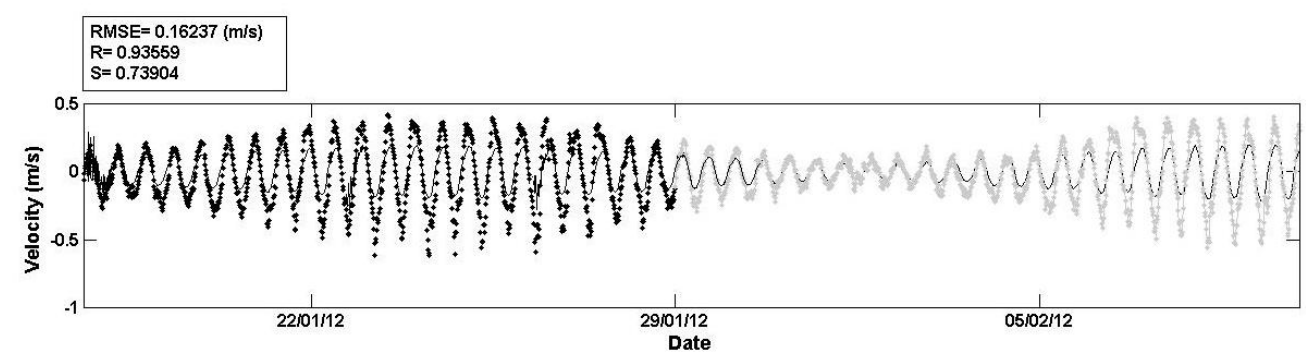

Figura 13: Velocidad Este (BR):Linea-Modelado y Puntos-Observado

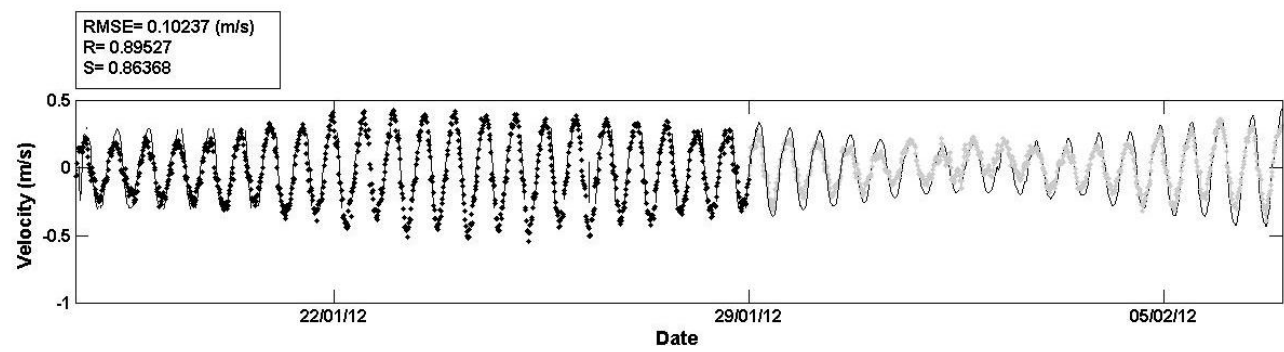

Figura 14: Velocidad Este (BF):Linea-Modelado y Puntos-Observado 


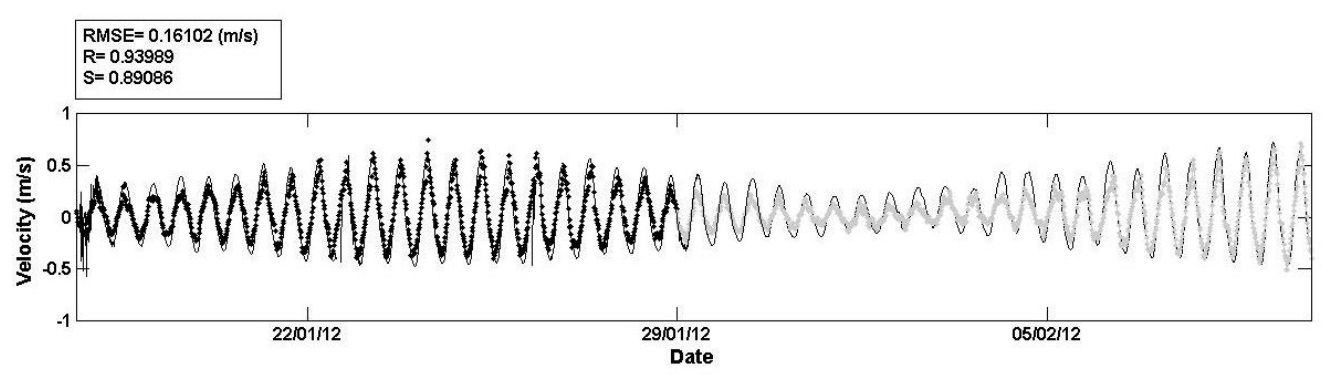

Figura 15: Velocidad Norte (BR):Linea-Modelado y Puntos-Observado

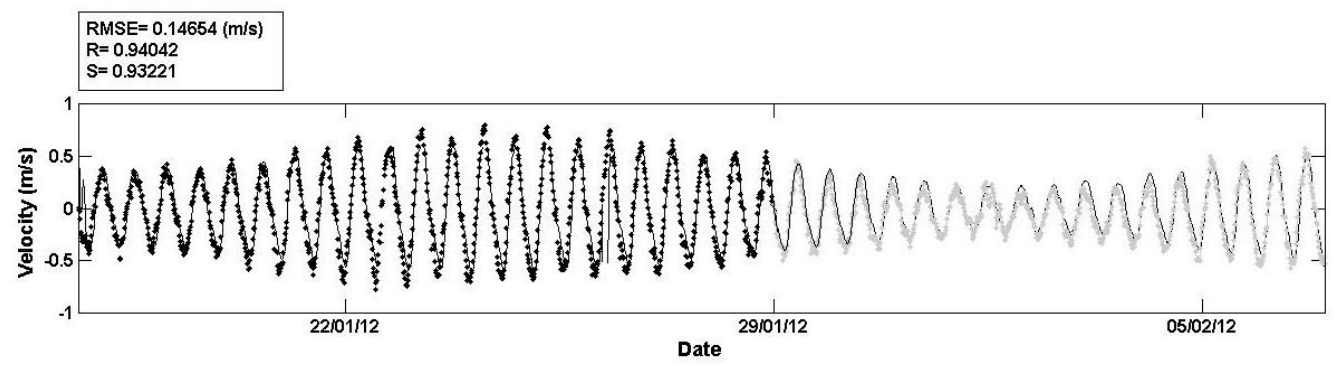

Figura 16: Velocidad Norte (BF):Linea-Modelado y Puntos-Observado
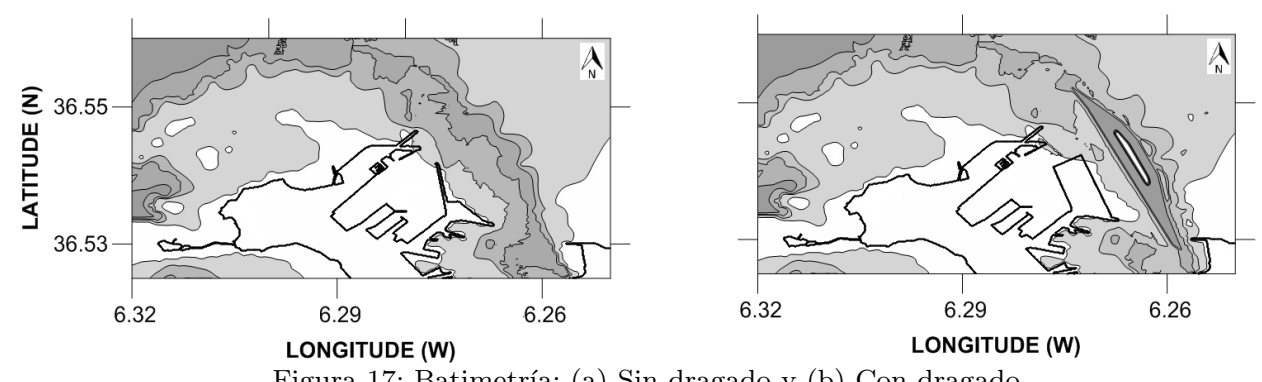

Figura 17: Batimetría: (a) Sin dragado y (b) Con dragado 

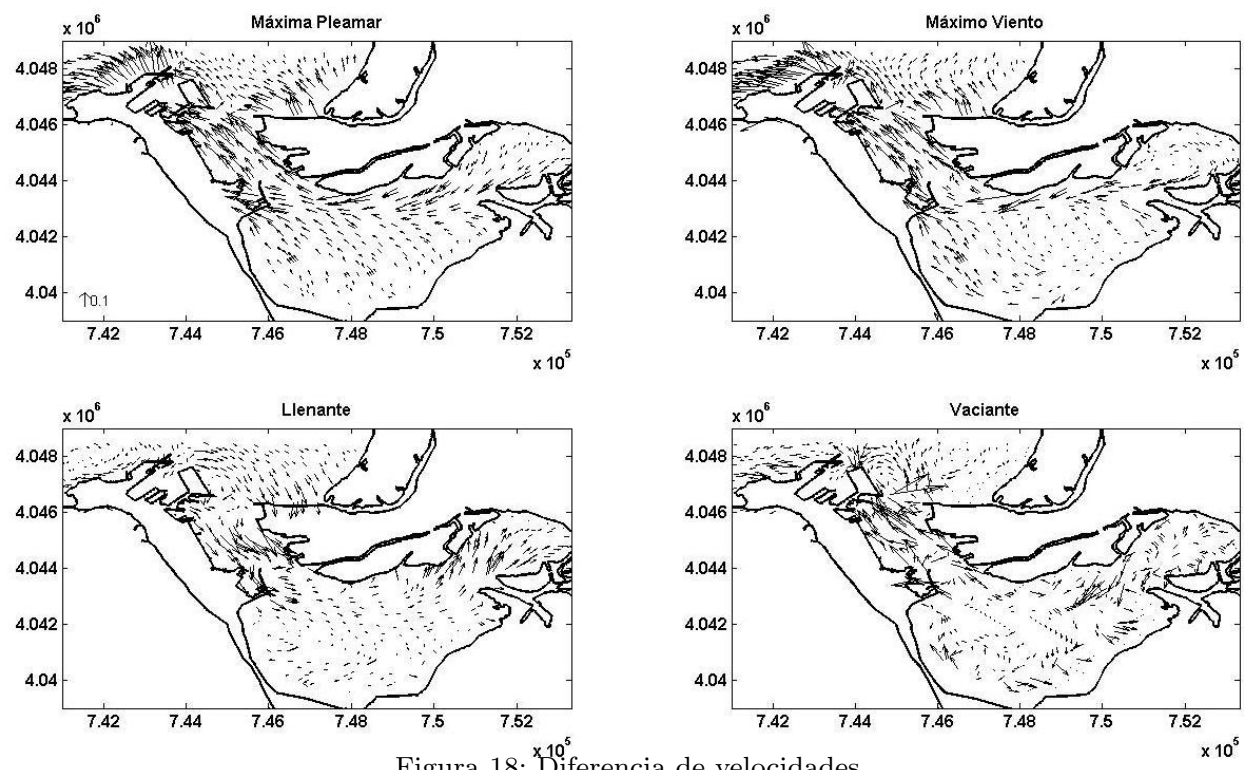

Figura 18: $\stackrel{\times 10^{5}}{\text { Diferencia de velocidade }}$

la batimetría y topografía, así como a la rugosidad y a los parámetros de calibración primaria: (1) 18 constituyentes de marea de RSP, (2) $\mathrm{U}=80$ y $\mathrm{V}=60$ como coeficientes de rugosidad de Chézy y (3) 0.005 como coeficiente de arrastre de viento. La correlación de los valores modelados con respecto a los observados osciló entre $\mathrm{RMSE}=0.97-0.99$ para el nivel del mar, y entre $\mathrm{RMSE}=0.73-0.95$ para las velocidades.

- Para validar el comportamiento del modelo, se han simulado otras fechas próximas a la de calibración (29/01/12 al 10/02/12) con las mismas variables definidas anteriormente. Los resultados fueron muy similares a los observados. Tras unir las dos series temporales simuladas se obtuvo una correlación de 0.89-0.98.

- Se ha empleado el modelo para simular el efecto del dragado que se realizará para la nueva ampliación del Puerto. Se han simulado las condiciones de máximo nivel y viento medidos durante la campaña, así como una situación de máxima velocidad de llenante y vaciante. Los resultados muestran que las partes más afectadas por el dragado son la parte externa de la Bahía y la parte superior del canal.

Las futuras líneas de investigación son:

- Analizar la hidrodinámica inducida por el oleaje, tanto a partir de los datos como aplicando el modelo SWAN.

- Simular otros escenarios de forzamiento (p.ej. vientos extremos).

- Analizar posibles correlaciones entre los distintos forzamientos.

- Analizar las medidas anómalas.

- Validar el modelo con otros periodos de tiempo.

\section{Agradecimientos}

Quisiera dar las gracias a los tutores (Dr. Miguel Ortega y Alejandro López) por su dedicación, imprescindible para llevar a cabo este trabajo.También agradecer al Grupo de Dinámica de Fluidos Ambientales (CEAMA) por su ayuda e implicación, en especial a Dr. Manolo Díez Minguito, Dr.Asunción Baquerizo e Inmaculada Ruiz por su disponibilidad para resolver los problemas que iban surgiendo.

Agradezco a la Consejería de Educación y Ciencia de la Junta de Andalucía la concesión de de la Beca de Formación de Personal investigador correspondiente al proyecto de excelencia, con convocatoria 2010, y a la Autoridad Portuaria de Cádiz por la ayuda prestada en la campaña de campo.

Por último gracias a mi familia y a José, por todo su apoyo y paciencia que han sido claves durante este tiempo. 


\section{Referencias}

Alvarez, O., Izquierdo, A., Tejedor, B., Mañares, R., 1999 The Influence of Sediment Load on Tidal Dynamics, a Case Study : Cádiz Bay. October 48, 439-450.

Álvarez, O., Tejedor, B., Tejedor, L., Kagan, B.A., 2003. A note on sea-breeze-induced seasonal variability in the $\mathrm{K} 1$ tidal constants inCádiz Bay, Spain. Estuarine, Coastal and Shelf Science 58, 805-812.

Benavente, J., Del Río, L., Gracia, F.J., MartÄnez, J.A., 2006. Coastal flooding hazard related to storms and coastal evolution in Valdelagrana spit ( Cadiz Bay Natural Park, SW Spain ). Continental Shelf Research 26, 10611076.

Carballo, R., 2009. Numerical Modelling of the Currents in the Rí de Muros y Noya and thair Energy Potential. Ph. D. Thesis, USC, Santiago de Compostela (Spain). Ph.D. thesis.

De la Paz, M., Gómez-Parra, A., Forja, J., 2008. Tidal-toseasonal variability in the parameters of the carbonate system in a shallow tidal creek influenced by anthropogenic inputs, Rio San Pedro ( SW Iberian Peninsula). Continental Shelf Research 28, 1394- 1404.

Elias, E., 2006. Morphodynamics of Texel Inlet. Ph.D. Thesis, Delft University of Technology, Faculty of Civil Engineering and Geosciences. Delft University Press (Delft). Ph.D. thesis.

Elias, E., Hansen, J., 2012. Understanding processes controlling sediment transports at the mouth of a highly energetic inlet system (San Francisco Bay, CA). In Press. Marine Geology .

Ferrón, S., Ortega, T., Forja, J.M., 2009. Benthic fluxes in a tidal salt marsh creek affected by fish farm activities : Río San Pedro ( Bay of Cádiz, SW Spain ). Marine Chemistry 113, 50-62.

Gutiérrez-mas, J.M., López-arroyo, J., Morales, J.A., 2009. Recent marine lithofacies in Cadiz Bay ( SW Spain ) Sequences, processes and control factors. Sedimentary Geology 218, 31-47.

Iglesias, G., Carballo, R., 2009. Seasonality of the circulation in the Ría de Muros (NW Spain). Journal of Marine Systems 78, 94-108.

Iglesias, G., Sánchez, M., Carballo, R., Fernández, H., 2012. The TSE index e A new tool for selecting tidal stream sites in depth-limited regions. Renewable Energy 48, 350-357.

Kagan, B.A., Alvarez, O., Izquierdo, A., 2005. Weak windwave / tide interaction over fixed and moveable bottoms : a formulation and some preliminary results. Continental Shelf Research 25, 753-773.

Kagan, B.A., Álvarez, O., Izquierdo, A., Mañanes, R., Tejedor, B., Tejedor, L., 2003. Weak wave / tide interaction in suspended sediment-stratified flow : a case study. Estuarine, Coastal and Shelf Science 56, 989-1000.

Kagan, B.A., Tejedor, L., Álvarez, O., Izquierdo, A., Tejedor, B., Mañanes, R., 2001. Weak wave - tide interaction formulation and its application to Cádiz Bay. Continental Shelf Research 21, 697-725.

Lesser, G.R., 2009. An approach to medium-term coastal morphological modelling. Ph. D. Thesis, UNESCO-IHE Institute for Water Education, Delft (the Netherlands). Ph.D. thesis.

Lesser, G.R., Roelvink, J.A., Kester, J.A.T.M.V., Stelling, G.S., 2004. Development and validation of a threedimensional morphological model. Coastal Engineering $51,883-915$.
Nielsen, P., 1986. Analysis of Natural Waves by Local Approximations.

Pawlowicz, R., Beardsley, B., Lentz, S., 2002. Classical tidal harmonic analysis including error estimates in MATLAB using T_TIDE. Computers \& Geosciences 28, 929-937.

Rodi, W., 1984. Turbulence model and their application in hydraulics, State-of-the-art paper article sur l'etat de connaissannce. Paper presented by the IAHR-Section on Fundamentals of Division II; Experimental and Mathematical Fluid Dynamics, The Netherlands.

Tejedor, B., G.E.A.A.V.J.R.A. (Ed.), 1997. Estudio de las anomalías diurnas de las corrientes en la Bahía de Cádiz. volume of , IV Jornadas Españolas de Ingeniería de Costas y Puertos, Servicio de Publicaciones de la Universidad Politécnica de Valencia-98.2125, pp. 87-99. , . .

Van der Wegen, M., Dastgheib, A., Roelvink, J.A., 2010. Morphodynamic modeling of tidal channel evolution in comparison to empirical PA relationship. Coastal Engineering 57, 827-837. 